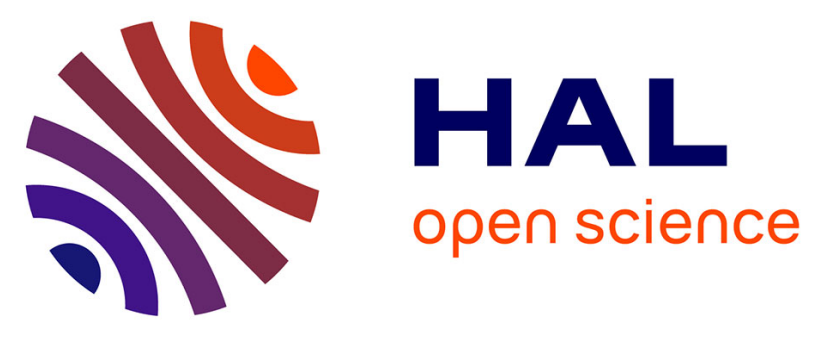

\title{
Impact de l'homogénéisation du lait maternel pasteurisé sur les cinétiques de digestion gastrique chez le nouveau-né prématuré
}

\author{
Samira Cassia de Oliveira, Olivia Ménard, Amandine Bellanger, Patrick \\ Pladys, Yann Le Gouar, Gwenaele Henry, Emelyne Dirson, Florence \\ Rousseau, Frédéric Carriere, Didier Dupont, et al.
}

\section{To cite this version:}

Samira Cassia de Oliveira, Olivia Ménard, Amandine Bellanger, Patrick Pladys, Yann Le Gouar, et al.. Impact de l'homogénéisation du lait maternel pasteurisé sur les cinétiques de digestion gastrique chez le nouveau-né prématuré. Journées Francophones de Nutrition, Nov 2016, Montpellier, France. 2016. hal-01404557

\author{
HAL Id: hal-01404557 \\ https://hal.science/hal-01404557
}

Submitted on 3 Jun 2020

HAL is a multi-disciplinary open access archive for the deposit and dissemination of scientific research documents, whether they are published or not. The documents may come from teaching and research institutions in France or abroad, or from public or private research centers.
L'archive ouverte pluridisciplinaire HAL, est destinée au dépôt et à la diffusion de documents scientifiques de niveau recherche, publiés ou non, émanant des établissements d'enseignement et de recherche français ou étrangers, des laboratoires publics ou privés. 


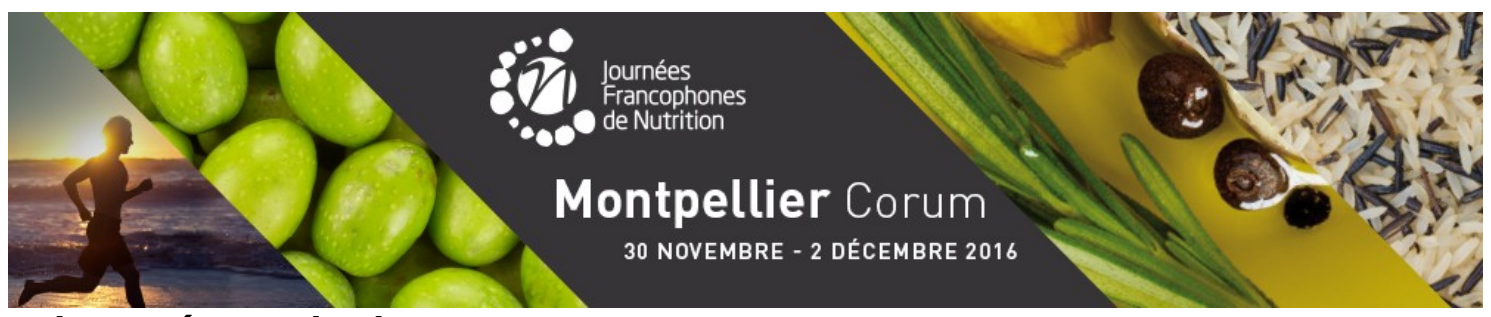

Pédiatrie et néonatologie

JFN2016/1202

Impact de l'homogénéisation du lait maternel pasteurisé sur les cinétiques de digestion gastrique chez le nouveau-né prématuré

Samira De Oliveira ${ }^{1}$, Olivia Ménard ${ }^{2}$, Amandine Bellanger $^{3}$, Patrick Pladys ${ }^{3}$, Yann Le Gouar $^{2}$, Gwénaële Henry², Emelyne Dirson ${ }^{4}$, Florence Rousseau² ${ }^{2}$ Frédéric Carrière ${ }^{5}$, Didier Dupont ${ }^{2}$, Claire Bourlieu ${ }^{2}$, Amélie Deglaire $^{* 2}$

${ }^{1}$ INRA - UMR 1253 STLO, ${ }^{2}$ Agrocampus Ouest, INRA - UMR 1253 STLO, ${ }^{3}$ Service Néonatalogie, ${ }^{4}$ Unité pédiatrique, CHU Rennes, Rennes, ${ }^{5} \mathrm{CNRS}$, Aix Marseille Université, UMR7282 Enzymologie Interfaciale \& Physiologie de la Lipolyse, Marseille, France

Discipline : Clinique

Présentation préférée : Communication orale

Introduction et but de l'étude : Assurer une croissance et un développement optimal chez les nouveau-nés prématurés est une priorité (WHO, 2015). Contrairement aux formules infantiles, le lait maternel permet de limiter les entérocolites nécrosantes, mais doit être supplémenté pour répondre aux besoins nutritionnels du prématuré. Cette stratégie est largement débattue. Une alternative pourrait être l'homogénéisation du lait maternel pasteurisé afin d'augmenter sa digestibilité lipidique (Thomaz, Goncalves, \& Martinez, 1999). L'homogénéisation, qui fragmente les globules gras en gouttelettes, conduit à l'augmentation de la surface disponible pour les lipases (Bourlieu et al., 2015). L'objectif était d'évaluer l'impact de l'homogénéisation de lait pasteurisé sur ses cinétiques d'hydrolyse gastrique et sa déconstruction en phase gastrique chez le prématuré.

Matériel et méthodes : 8 enfants prématurés hospitalisés nourris par sonde gastrique ont participé à cette étude randomisée (NCT02112331). Sur 6 jours consécutifs, chaque enfant recevait chaque jour du lait maternel pasteurisé (LMP) et pasteurisé homogénéisé $(\mathrm{LMP}+\mathrm{H})$. Les effluents gastriques étaient collectés à jeun puis à 35, 60 ou 90 min, chaque temps étant collecté en double. Après mesure du volume et $\mathrm{pH}$, les effluents étaient analysés par granulométrie laser et microscopie confocale, puis par électrophorèse (SDSPAGE) pour le suivi des protéines et par chromatographie pour le suivi lipidique.

Concernant les statistiques (logiciel R), un modèle linéaire mixte était utilisé avec comme facteurs : temps, repas, sujet et sujet $x$ repas, et éventuellement après sélection de modèle : jour de prélèvement ou poids de l'enfant, teneur en lipides ou protéines.

Résultats et Analyse statistique : L'homogénéisation du lait maternel pasteurisé a conduit à une réduction significative de taille des particules d'un facteur 10 [mode de la distribution (moy $\pm \mathrm{ET}$ ) : $7.0 \pm 0.9$ (LMP) vs. $0.8 \pm 0.3-0.16 \pm 0 \mu \mathrm{m}(\mathrm{LMP}+\mathrm{H})]$ et une augmentation significative de la surface spécifique d'un facteur $6[4.1 \pm 1.2$ (LMP) vs 25.5 $\pm 3.8 \mathrm{~m}^{2} / \mathrm{g}$ de lipide $\left.(\mathrm{LMP}+\mathrm{H})\right]$. L'homogénéisation accélérait significativement la lipolyse instantanée $(\mathrm{LMP}+\mathrm{H}: 10.9 \pm 1.8,12.7 \pm 2.3$ et $15.0 \pm 7.1 \%$; LMP : $8.5 \pm 2.7,9.6 \pm 2.6$ et $13.8 \pm 5.2 \%$ à 35, 60 et 90 min, respectivement) mais n'avait pas d'impact sur la protéolyse. Un ralentissement significatif de la vidange gastrique du repas était observé pour LMP+H avec un temps de demi-vidange de 38 min contre 30 min pour LMP. II n'y avait pas de différence significative d'acidification gastrique entre LMP et LMP+H.

Conclusion : L'impact de l'homogénéisation du lait maternel pasteurisé sur l'augmentation du degré de lipolyse gastrique a été démontré in vivo, en parallèle d'un 
ralentissement de la vidange gastrique. L'impact global sur la croissance du prématuré reste à évaluer dans une étude clinique randomisée plus large.

Références : Bourlieu et al. (2015). Food Chemistry, 182, 224-235.

Thomaz et al. (1999). Nutrition Research, 19, 483-492.

WHO. (2015).

Conflits d'intérêts: Aucun conflit à déclarer 